\title{
Physiological and psychological effects of walking on young males in urban parks in winter
}

\author{
Chorong Song ${ }^{1}$, Dawou Joung ${ }^{2}$, Harumi Ikei ${ }^{1}$, Miho Igarashi', Mariko Aga', Bum-Jin Park², Masayuki Miwa', \\ Michiko Takagaki ${ }^{1}$ and Yoshifumi Miyazaki ${ }^{*}$
}

\begin{abstract}
Background: Interaction with nature has a relaxing effect on humans. Increasing attention has been focused on the therapeutic effects of urban green space; however, there is a lack of evidence-based field research. This study provided scientific evidence supporting the physiological and psychological effects of walking on young males in urban parks in winter.
\end{abstract}

Findings: Subjects (13 males aged $22.5 \pm 3.1$ years) were instructed to walk predetermined 15 -minute courses in an urban park (test) and in the city area (control). Heart rate and heart rate variability (HRV) were measured to assess physiological responses. The semantic differential (SD) method, Profile of Mood States (POMS), and State-Trait Anxiety Inventory (STAI) were used to determine psychological responses.

Heart rate was significantly lower and the natural logarithm of the high frequency component of HRV was significantly higher when walking through the urban park than through the city area. The results of three questionnaires indicated that walking in the urban park improved mood and decreased negative feelings and anxiety.

Conclusions: Physiological and psychological data from this field experiment provide important scientific evidence regarding the health benefits of walking in an urban park. The results support the premise that walking in an urban park has relaxing effects even in winter.

Keywords: Urban green space, Walking, Winter, Stress, Physiological relaxation, Heart rate, Heart rate variability, Semantic differential method, Profile of mood states, State-trait anxiety inventory

\section{Findings}

\section{Background}

It is empirically known that interaction with nature has a relaxing effect. As the interest in health promotion and quality of life has increased, attention has been focused on the role of nature in promoting human health and well-being.

Humans evolved into what they are today after the passage of six or seven million years [1]. Therefore, more than $99.99 \%$ of human evolutionary history was spent in the natural environment. Urbanization can be defined as a post-industrial revolutionary development. Through centuries of evolution within the natural environment, humans adapted to nature [2,3]. This human tendency

\footnotetext{
* Correspondence: ymiyazaki@faculty.chiba-u.jp

${ }^{1}$ Center for Environment, Health and Field Sciences, Chiba University, Chiba, Japan

Full list of author information is available at the end of the article
}

to be close to nature implies that contact with nature may be an important component of well-being [4].

Many studies have demonstrated significant positive psychological and physiological benefits of interaction with nature. Interaction with nature aids recovery after attentional fatigue [5] and stress [2] and improves emotional state [6]. Studies of the physiological effects of relaxation in forest environments have tested parameters such as cerebral activity in the prefrontal area [7], pulse rate $[8-10]$, blood pressure $[9,11]$, heart rate variability (HRV) $[8,10,11]$, salivary cortisol concentration [7-10], and natural killer (NK) cell activity $[12,13]$. Many results based on scientific evidence have been reported [14-16].

However, in modern society, interaction with nature such as forests is difficult. Recently, increasing attention has been focused on the role of urban green spaces such as urban parks that provide a natural environment close to most people in modern society. Recent demographic

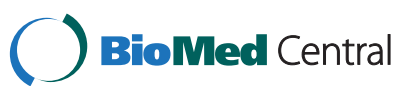


research found a positive association between exposure to urban green space and perceived general health of residents [17]. Living in areas with walkable green spaces positively influenced the longevity of urban senior citizens independent of their age, sex, marital status, baseline functional status, and socioeconomic status [18].

However, there is a lack of evidence-based research on the therapeutic effects of urban green space. Many scientists have emphasized the importance of field research [19]. Because the therapeutic effects of nature occur without conscious thought, these effects must be clarified in physiological field studies [3]. Furthermore, although we conducted similar experiments during summer [20], there are no experimental examples that can verify the physiological effects during winter.

The aim of this study was to provide scientific evidence supporting the physiological and psychological effects of walking in urban parks in winter.

\section{Methods}

The field experiment was performed in November 2012 in Kashiwanoha Park (hereinafter referred to as the urban park) in Chiba, Japan. As a control, the city area around the urban park (hereinafter referred to as the city area) was selected (Figure 1). The weather on the day of the experiment was sunny, and the average temperature, humidity, and intensity of illumination in the urban park were $13.8^{\circ} \mathrm{C}, 50.9 \%$, and $7,930 \mathrm{~lx}$, respectively, while those in the city area were $14.0^{\circ} \mathrm{C}, 52.1 \%$, and $8,430 \mathrm{~lx}$, respectively. In addition, the trees in the park had either lost their leaves or the leaves had turned red or yellow, but there was no snow.

Thirteen Japanese male university students $(22.5 \pm 3.1$ years old) participated in this experiment. Each subject walked in the urban park or city area for 15 minutes. None reported a history of physical or psychiatric disorders. This study was performed according to the regulations of the Ethics Committee of the Center for Environment, Health and Field Sciences, Chiba University, Japan.

Heart rate and HRV were measured to assess physiological responses. HRV, which is often used to assess human autonomic activity, was measured using a portable electrocardiograph (Activtracer AC-301A, GMS, Tokyo, Japan). HRV data were obtained at various frequencies using an HRV software tool (MemCalc/Win, GMS). For real-time HRV analysis using the maximum entropy method, inter-beat (R-R) intervals were obtained continuously. In this study, the two major HRV spectral components, low frequency (LF; 0.04 to $0.15 \mathrm{~Hz}$ ) and high frequency (HF; 0.15 to $0.40 \mathrm{~Hz}$ ) band variance, were calculated. The LF/HF ratio in R-R interval variability was also assessed. HF components can be a general indication of parasympathetic nervous activity, and the LF/HF ratio can be used as an index of sympathetic nervous activity $[21,22]$. To normalize the distribution of HRV parameters, we used natural logarithmic transformed values for the analysis [23]. The heart rate and HRV data, which were collected at 1 minute intervals at each experimental location, were compared based on the average for 15 minutes.

Three different questionnaires were used to investigate psychological responses. The questionnaires were completed after walking at each experimental site. Evaluation using semantic differential (SD) method [24] was performed using three pairs of adjectives on seven scales, including 'comfortable to uncomfortable,' 'natural to artificial', and 'relaxed to awakening'. The Profile of Mood State (POMS) data were analyzed using the following six subscales: 'tension-anxiety', 'depression', 'anger-hostility', 'vigor,' 'fatigue', and 'confusion'. In the POMS test, a short form with 30 questions was used to reduce the burden on the subjects $[25,26]$. The State-Trait Anxiety Inventory (STAI) [27] was used to evaluate anxiety.

We performed a within-subject experiment, and to eliminate the effect of the order of sites walked, two subjects were paired. One subject walked in the urban park first and in the city area later, while the other walked in the city area first and then in the urban park. There was no difference in the physiological index before the start of each walk between the two environments. After walking, the subjects returned to the waiting room and completed questionnaires. They rested for approximately 20 minutes and repeated the experiment by walking at the alternate
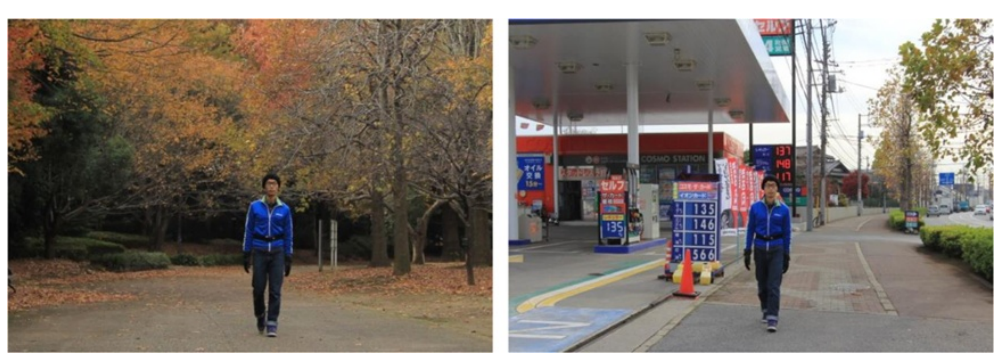

Figure 1 Experimental scene of urban park (left) and city area (right). 
experimental site. In addition, there was no difference in the walking speed between the two environments.

All data were shown as mean \pm standard deviation. A paired $t$-test was used to compare the differences in the mean physiological data scores over a period of $15 \mathrm{mi}-$ nutes while walking in the urban park and city area. Wilcoxon signed-rank test was used to analyze differences in the psychological indices after walking between the two environments. Statistical analysis was performed using SPSS 20.0 (SPSS Inc, Chicago, IL, USA). A onesided test was used in this study. In all cases, values of $P<0.05$ were considered statistically significant.

\section{Results and discussion}

In the comparison of physiological indices between the urban park and the city area, key differences were observed. Subjects' heart rates were significantly lower (4.4\%) after walking in the urban park $(98.4 \pm 0.9 \mathrm{bpm})$ than after walking in the city area $(102.9 \pm 1.1 \mathrm{bpm} ; P<0.05$; Figure 2). When the results of HRV data were compared, a significant difference was found in the natural logarithm of HF $(\ln (\mathrm{HF}))$, which is a marker of parasympathetic nervous activity, between the two environmental stimuli. The urban park $\left(4.61 \pm 1.25 \mathrm{msec}^{2}\right)$ showed a $21.6 \%$ higher value than the city area $\left(3.79 \pm 1.16 \mathrm{msec}^{2}\right.$; $P<0.05$; Figure 3$)$. In the comparison of $\ln (\mathrm{LF} / \mathrm{HF})$ values, which is a marker of sympathetic nervous activity, no significant difference between the two environments was found. However, a trend towards lower values in the urban park $(1.06 \pm 1.01$ ratio) compared with the city area

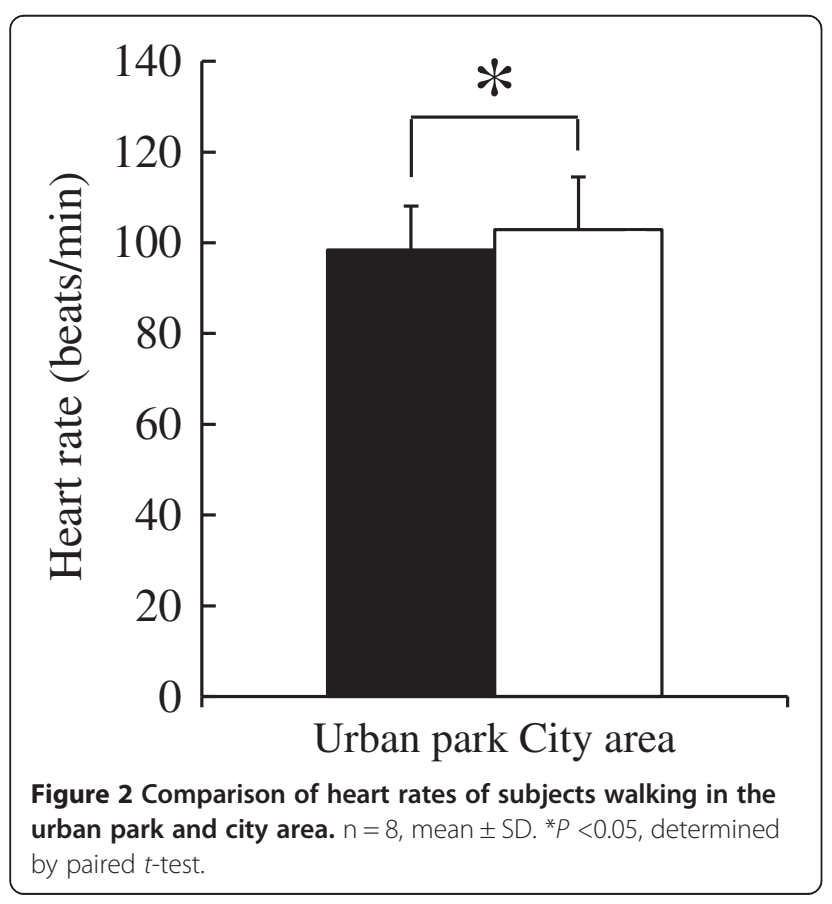

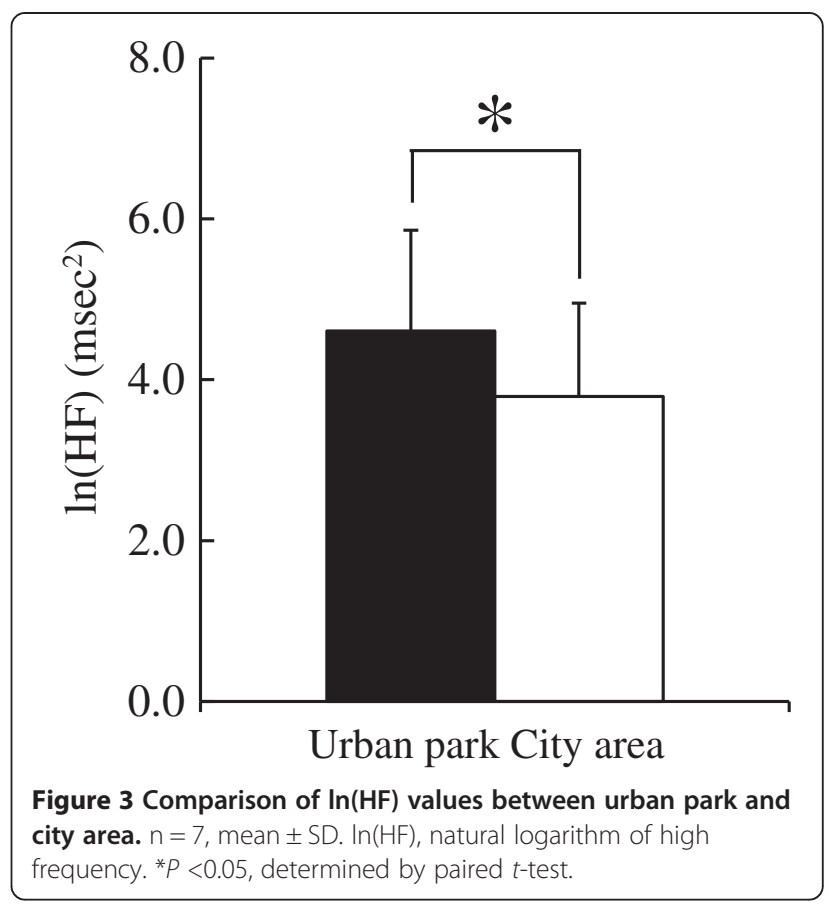

$(1.40 \pm 1.00$ ratio $)$ was detected $(P=0.06)$. These physiological reactivity results correlated partly with those reported by previous studies related to forest therapy [8,10,11,14-16], supporting the hypothesis that urban parks have similar health benefits to natural environments.

The results of the analysis of psychological responses revealed notable differences between the two environments. In the comparison of the SD scores, significantly higher scores were observed in the urban park for the following three adjectives: 'comfortable', 'natural', and 'relaxed' compared with the city area $(P<0.01$; Figure 4$)$. Significant differences were also detected in the results of the POMS test (Figure 5). The score for the negative subscale 'tension-anxiety' was significantly lower after walking in the urban park compared with the city area $(P<0.01)$. Conversely, the positive mood state for 'vigor' was significantly higher in the urban park but not in the city area $(P<0.01)$. For the other subscales ('depression', 'anger-hostility', 'fatigue', and 'confusion'), no significant differences were observed. In the results of analysis of state anxiety using STAI, the score was $18.2 \%$ lower in the urban park ( $37.3 \pm 8.7$ scores) compared with the city area (45.6 \pm 7.1 scores; $P<0.05$; Figure 6).

Based on the analysis of these three psychological indices, subjects felt more comfortable, natural, relaxed, and vigorous when walking in the urban park than in the city area. In addition, the negative emotions and anxiety levels were significantly lower. These results on the psychological benefits of walking in the urban park are partly consistent with previous findings related to forest therapy [8-11]. 
Urban park $\square$ City area

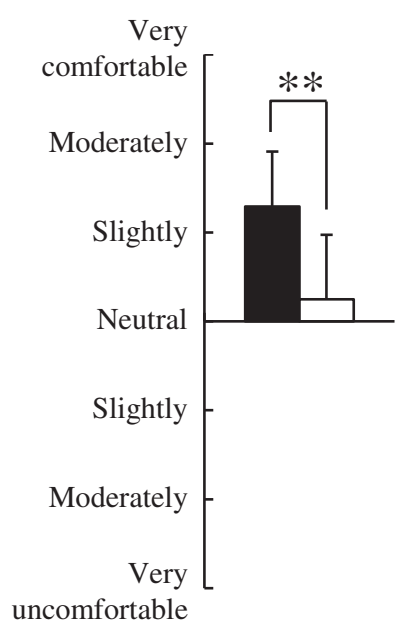

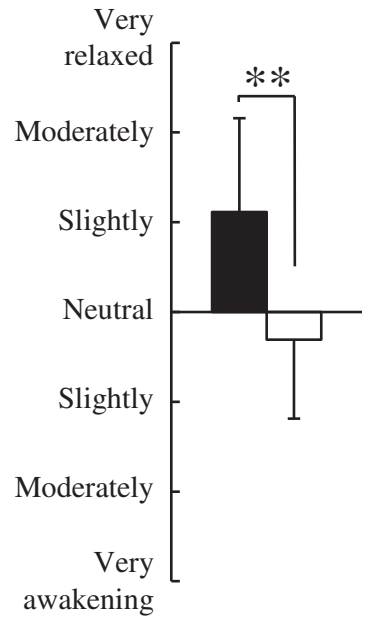

awakening

Figure 4 Comparison of subjective scoring for comfortable, natural, and relaxed feelings between the two environments. $n=13$, mean $\pm \mathrm{SD}$. ${ }^{* *} P<0.01$, determined by Wilcoxon signed-rank test.

For those who desire a higher quality of life, scientific evidence about the relaxation effects of urban green space must be accumulated, as these are the natural environments most accessible to people in modern society.

However, these results cannot be extrapolated to the female population and people of different age groups or ethnicities, because only thirteen young male adults participated in this study. To generalize the findings, further evidence-based studies on a large sample including various subject groups is required.

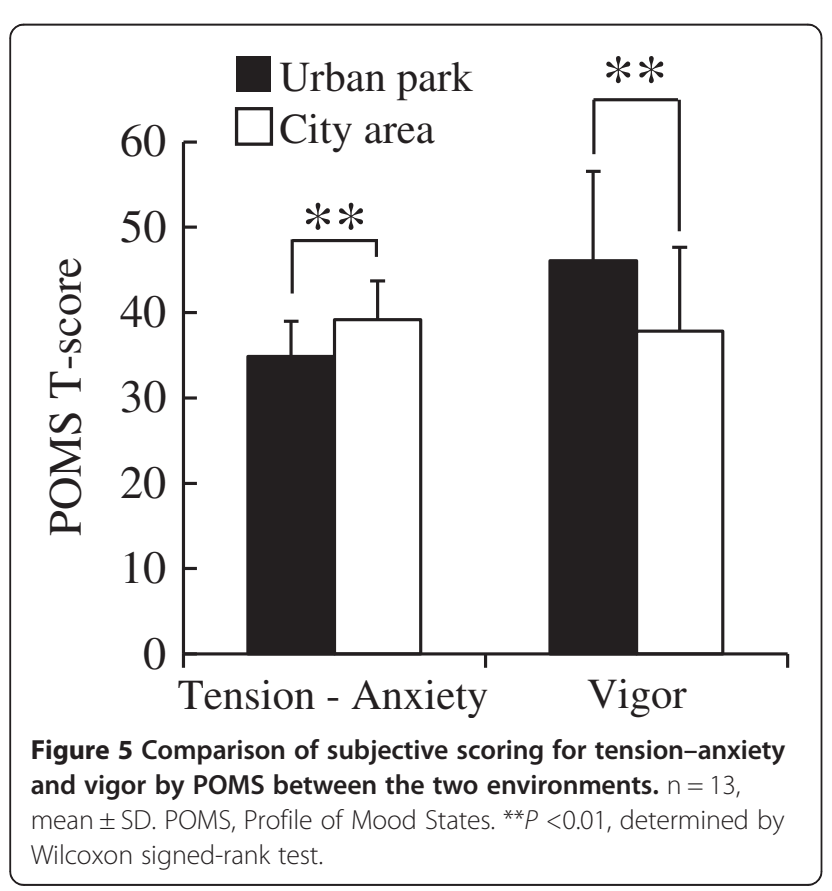

This study may contribute toward progress in the area of physiological anthropology because identifying the physiological effects of the natural environment is an important issue.

\section{Conclusion}

These findings provide important scientific evidence of the health benefits of walking in urban parks. The

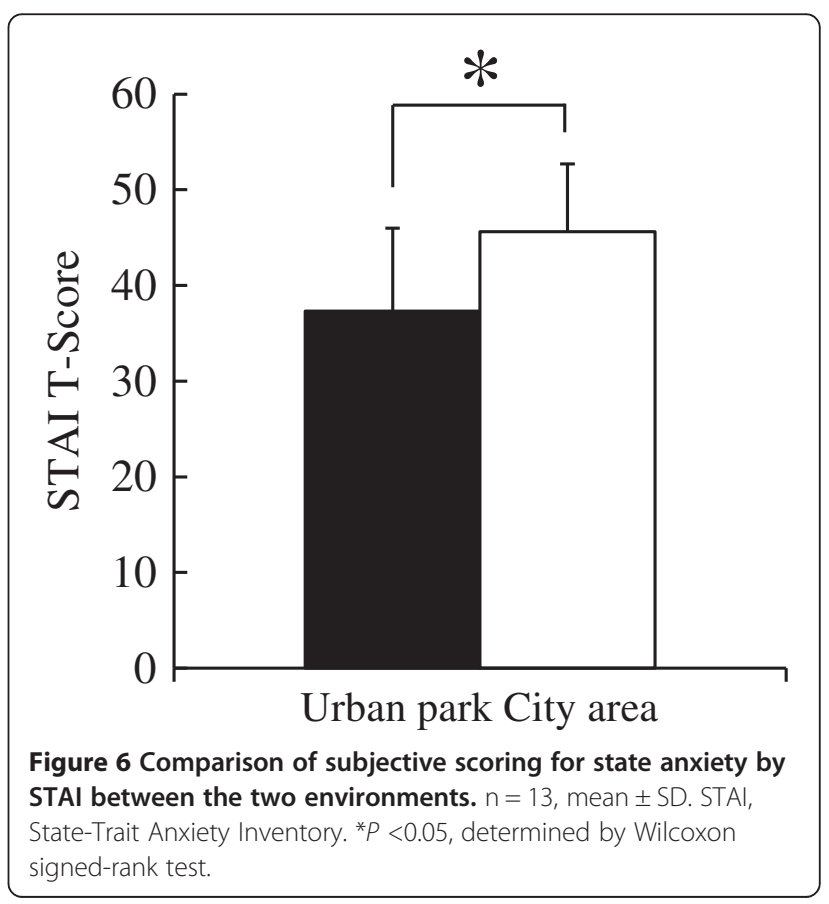


results support the premise that walking in urban parks has relaxing effects even in winter.

\author{
Abbreviations \\ HF: High frequency; HRV: Heart rate variability; LF: Low frequency; NK: Natural \\ killer; POMS: Profile of mood states; SD: Semantic differential; STAl: State-trait \\ anxiety inventory.
}

\section{Competing interests}

The authors declare that they have no competing interests.

\section{Authors' contributions}

CS contributed to the study design, data acquisition, statistical analysis, interpretation of the results, and manuscript preparation. DJ, $\mathrm{Hl}, \mathrm{Ml}$, and MA were involved with data acquisition and statistical analysis. BP conceived and designed the study. MM and MT participated in the design of the study and carried out interpretation of data. YM had an important role in the research, particularly in experimental design, data acquisition, and manuscript preparation. All authors contributed to the preparation and are responsible for the final editing and approval of the manuscript.

\section{Acknowledgements}

This study was performed by permission of the Corporation for Urban Enhancement of Chiba Prefecture, Japan. We appreciate their co-operation. For valuable contributions in the data collection phase of this study, we are grateful to Dr Yuko Tsunetsugu of the Forestry and Forest Products Research Institute, Japan.

\section{Author details}

${ }^{1}$ Center for Environment, Health and Field Sciences, Chiba University, Chiba, Japan. ${ }^{2}$ Department of Environment and Forest Resources, Chungnam National University, Daejeon, Korea.

Received: 3 April 2013 Accepted: 17 October 2013

Published: 29 October 2013

\section{References}

1. Brunet M, Guy F, Pilbeam D, Mackaye HT, Likius A, Ahounta D, Beauvilain A, Blondel C, Bocherens H, Boisserie JR, Bonis L, Coppens Y, Dejax J, Denys C, Duringer $P$, Eisenmann V, Fanone G, Fronty P, Geraads D, Lehmann T, Lihoreau F, Louchart A, Mahamat A, Merceron G, Mouchelin G, Otero O, Campomanes PP, Leon MPD, Rage JC, Sapanet M, et al: A new hominid from the Upper Miocene of Chad, Central Africa. Nature 2002, 418:141-151.

2. Ulrich RS, Simons RF, Losito BD, Fiorito E, Miles MA, Zelson M: Stress recovery during exposure to natural and urban environments. J Environ Psychol 1991, 11:201-230

3. Miyazaki Y, Park BJ, Lee J: Nature therapy. In Designing our Future: Local Perspectives on Bioproduction, Ecosystems and Humanity. Edited by Osaki M, Braimoh A, Nakagami K. New York, NY: United Nations University Press; 2011:407-412.

4. Frumkin $\mathrm{H}$ : Beyond toxicity, human health, and the natural environment. Am J Prev Med 2001, 20:234-240.

5. Kaplan R, Kaplan S: The Experience of Nature: A Psychological Perspective. New York, NY: Cambridge University Press; 1989.

6. Shin WS, Yeoun PS, Yoo RW, Shin CS: Forest experience and psychological health benefits: the state of the art and future prospect in Korea. Environ Health Prev Med 2010, 15:38-47.

7. Park BJ, Tsunetsugu Y, Kasetani T, Hirano H, Kagawa T, Sato M, Miyazaki Y: Physiological effects of Shinrin-yoku (taking in the atmosphere of the forest)-using salivary cortisol and cerebral activity as indicators. J Physiol Anthropol 2007, 26:123-128.

8. Park BJ, Tsunetsugu Y, Kasetani T, Morikawa T, Kagawa T, Miyazaki Y: Physiological effects of forest recreation in a young conifer forest in Hinokage town, Japan. Silva Fenn 2009, 43:291-301.

9. Lee J, Park BJ, Tsunetsugu Y, Kagawa T, Miyazaki Y: Restorative effects of viewing real forest landscapes, based on a comparison with urban landscapes. Scand J Forest Res 2009, 24:227-234.

10. Lee J, Park BJ, Tsunetsugu Y, Ohira T, Kagawa T, Miyazaki Y: Effect of forest bathing on physiological and psychological responses in young Japanese male subjects. Public Health 2011, 125:93-100.
11. Tsunetsugu Y, Lee J, Park BJ, Tyrvainen L, Kagawa T, Miyazaki Y: Physiological and psychological effects of viewing urban forest landscapes assessed by multiple measurement. Landsc Urban Plan 2013, 113:90-93.

12. Li Q, Morimoto K, Kobayashi M, Inagaki H, Katsumata M, Hirata Y, Hirata K, Suzuki H, Li YJ, Wakayama Y, Kawada T, Park BJ, Ohira T, Matsui N, Kagawa T, Miyazaki Y, Krensky AM: Visiting a forest, but not a city, increases human natural killer activity and expression of anti-cancer proteins. Int J Immunopathol Pharmacol 2008, 21:117-127.

13. Li Q, Morimoto K, Kobayashi M, Inagaki H, Katsumata M, Hirata Y, Hirata K, Shimizu T, Li YJ, Wakayama Y, Kawada T, Ohira T, Takayama N, Kagawa T, Miyazaki Y: A forest bathing trip increases human natural killer activity and expression of anti-cancer proteins in female subjects. J Biol Regul Homeost Agents 2008, 22:45-55.

14. Park BJ, Tsunetsugu Y, Lee J, Kagawa T, Miyazaki Y: Forest medicine. In Effect of the Forest Environment on Physiological Relaxation Using the Results of Field Tests at 35 Sites Throughout Japan. Edited by Li Q. New York, NY: Nova Science Publishers; 2012:55-65.

15. Tsunetsugu Y, Park BJ, Miyazaki Y: Trends in research related to "Shinrin-yoku" (taking in the forest atmosphere or forest bathing) in Japan. Environ Health Prevent Med 2010, 15:27-37.

16. Lee J, Li Q, Tyrväinen L, Tsunetsugu Y, Park BJ, Kagawa T, Miyazaki Y: Nature therapy and preventive medicine. In Public Health-social and Behavioral Health. Edited by Maddock JR. Rijeka: InTech; 2012:325-350.

17. Maas J, Verheij RA, Groenewegen PP, Vries SD, Spreeuwenberg P: Green space, urbanity, and health: how strong is the relation? J Epidemiol Community Health 2006, 60:587-592.

18. Takano T, Nakamura $\mathrm{K}$, Watanabe M: Urban residential environments and senior citizens' longevity in megacity areas: the importance of walkable green spaces. J Epidemiol Community Health 2002, 56:913-918.

19. Groenewegen PG, van den Berg AE, DeVries S, Verheij RA: Vitamin G: effects of green space on health, well-being, and social safety. BMC Public Health 2006, 6:1-9.

20. Matsuba N, Lee J, Park BJ, Lee M, Song C, Miyazaki Y: Physiological effects of walking in Shinjuku Gyoen, a large-scale urban green area. Jpn J Physiol Anthropol 2011, 16:133-139.

21. Task Force of the European Society of Cardiology and the North American Society of Pacing and Electrophysiology: Heart rate variability: standards of measurement, physiological interpretation and clinical use. Circulation 1996, 93:1043-1065

22. Pagani M, Lombardi F, Guzzetti S, Rimoldi O, Furlan R, Pizzinelli P, Sandrone G Malfatto G, Dell'Orto S, Piccaluga E: Power spectral analysis of heart rate and arterial pressure variabilities as a marker of sympatho-vagal interaction in man and conscious dog. Circ Res 1986, 59:178-193.

23. Kobayashi H, Park BJ, Miyazaki Y: Normative references of heart rate variability and salivary alpha-amylase in a healthy young male population. J Physiol Anthropol 2012, 31:9.

24. Osgood CE, Suci GJ, Tannenbaum P: The Measurement of Meaning. Urbana, IL: University of Illinois Press; 1957

25. McNair D, Lorr M, Droppleman L: Profile of Mood States Manual. San Diego, CA: Educational and Industrial Testing Services; 1992.

26. Yokoyama K: POMS Shortened Version-Manual and Commentary on Cases. Tokyo: Kaneko Syoboh; 2005.

27. Hidano N, Fukuhara M, Iwawaki M, Soga S, Spielberger CD: State-Trait Anxiety Inventory- Form JYZ. Tokyo: Jitsumu-Kyoiku Syuppan; 2000.

doi:10.1186/1880-6805-32-18

Cite this article as: Song et al.: Physiological and psychological effects of walking on young males in urban parks in winter. Journal of Physiological Anthropology 2013 32:18 